\title{
Measuring Quality of Life in Epilepsy Surgery Patients
}

\author{
Samuel Wiebe and Paul A. Derry
}

\begin{abstract}
Treatment of chronic conditions often entails trade-offs between benefits and risks or side effects. This is of particular importance in epilepsy. Consequently, assessment of effectiveness must incorporate this trade-off as perceived by the recipient of the intervention, ie., the patient. Measurement of health related quality of life (HRQOL) aims at capturing such patient-centred effects of therapy. Conceptual aspects of HRQOL are described and issues relevant to outcomes of epilepsy surgery are addressed. Finally, practical aspects of measurement in the context of epilepsy surgery are discussed.
\end{abstract}

RÉSUMÉ: Mesure de la qualité de vie chez les patients qui ont subi une chirurgie pour l'épilepsie. Le traitement de maladies chroniques implique souvent des compromis entre les bénéfices et les risques ou les effets secondaires. C'est particulièrement important dans le traitement de l'épilepsie. L'évaluation de l'efficacité du traitement doit donc incorporer ces compromis, tels que perçus par le patient. L'évaluation de la qualité de vie reliée à la santé a pour but de cerner de tels effets du traitement chez le patient. Nous décrivons les aspects conceptuels de la qualité de vie reliée à la santé et discutons des questions pertinentes aux résultats de la chirurgie de l'épilepsie. Finalement, les aspects pratiques de l'évaluation dans le contexte de la chirurgie de l'épilepsie sont discutés.

Can. J. Neurol. Sci. 2000; 27: Suppl. 1 - S111-S115

The quality of one's life has multiple determinants, such as financial status, relationships, housing, recreation, health status, etc. Clinicians are concerned with health status as a determinant of QOL, or health-related quality of life. Conceptually, HRQOL can be defined as "a judgement of one's well-being based on consideration of physical, mental, social, and general health status." " Distinguishing between QOL and HRQOL is more important than a simple academic exercise. A recent appraisal of HRQOLmeasures used in clinical trials showed that instruments often failed to distinguish general QOL from HRQOL. ${ }^{2}$ Clearly, an instrument that asks about global QOL without specific reference to health, will tell us nothing about the health-related component of the estimate.

The common element to any HRQOLdefinition should be its ability to capture the patients'subjective perception of whatever health area we are interested in measuring. As aptly put by Gill and Feinstein, "HRQOL is a uniquely personal perception denoting the way that individual patients feel about their health status". ${ }^{2}$ The purpose is to review the available epilepsy-specific HRQOL instruments and the issues around its measurement in epilepsy surgery.

\section{TRADITIONAL OUTCOME MEASURES IN EPILEPSY}

Virtually all treatments in epilepsy embody trade-offs between seizure control and side effects. In refractory epilepsy, side effects may be severe and lifelong, since large amounts of drugs must be taken for life. Epilepsy surgery attempts to render patients seizure-free with minimum or no use of antiepileptic drugs (AED). However, assessment of surgical benefit and morbidity must enter the equation. Do patients prefer fewer seizures with more side effects, or vice-versa? Can surgery significantly improve the risk benefit ratio as compared to AEDs? What is the patient's experience of various treatments? These questions are directly related to patient care and wellbeing and can only be addressed by assessing HRQOL.

\section{Seizure frequency and severity:}

For decades, treatment outcome in epilepsy was measured in terms of seizure frequency. Subsequently, mainly in the context of AED trials, ${ }^{3}$ it was recognized that not all seizure types have the same impact on affected patients. The two most popular instruments to determine seizure severity are the Liverpool Seizure Severity Scale ${ }^{4}$ and the National Hospital Scale (NHS). ${ }^{5}$ Their development and accumulated experience with their use is summarized in the section "Seizures as indicators of therapeutic control" in this supplement.

From the Department of Clinical Neurological Sciences, University of Western Ontario, London, ON Canada

Reprint requests to: Samuel Wiebe, London Health Sciences Centre, University Campus, 339 Windermere Rd., London, Ontario N6A5A5 Canada 


\section{Psychosocial MEASUReS}

Crucial to formulation of disease specific HRQOL instruments was the early recognition that epilepsy inflicts serious psychosocial damage on its victims. This section reviews the conceptual evolution leading to the current notion of HRQOL.

\section{Initial efforts}

As early as the mid 1900s, Lennox ${ }^{6}$ theorized on the consequences of epilepsy on the patient's life, family, interictal behaviour and vocation.

Two decades later, Taylor ${ }^{7}$ proposed a revolutionary paradigm of epilepsy which still features in HRQOL models. It distinguishes between the disease (the biological manifestation), the illness (the experience of having the disorder), and the predicament (its effect on psychosocial status). Taylor's "illness" and "predicament" provide the conceptual framework for the subjective perception and impact of epilepsy in current HRQOL instruments.

For three decades, isolated psychological and functional instruments were used in a rather disorganized fashion, focusing on individual aspects of epilepsy such as driving, return to work, affective status, cognitive function, and personality profiles, without attempting a global assessment of HRQOL.

In 1980, using a psychometric approach, Dodrill ${ }^{8}$ developed the first comprehensive psychosocial measure specific for epilepsy patients, the 132-item, self-administered Washington Psychosocial Seizure Inventory (WPSI). The WPSI has a narrow scope, ${ }^{9}$ it may not discriminate among patients with mild and severe epilepsy ${ }^{10}$ and it does not detect small but clinically important changes in response to therapy. ${ }^{11}$ Nonetheless, this instrument facilitated transcultural research and paved the way for developing comprehensive HRQOL models in epilepsy.

\section{Interview-based assessment of patients'experiences}

To capture the patient's experience of epilepsy-related disability, Chaplin et al ${ }^{12}$ identified 14 areas of concern obtained by systematic patient interviews. Items reflect "the experience of patients with epilepsy, in their words, and unstructured by professional theory and models". ${ }^{13}$ The instrument has adequate cross sectional validity and test-retest reliability and is available in adult and children versions. However, experience with this tool is limited. For example, in a mailed survey, $89 \%$ of recently diagnosed patients had fear of seizures and 69\% had fear of stigma in employment. That concerns were less severe than in patients with chronic epilepsy supports the negative impact of chronicity on psychosocial function in this group of patients. ${ }^{14}$

\section{Current versus anticipated self-status or image}

Using a repertory grid technique, Kendrick et $\mathrm{al}^{15}$ asked patients with severe epilepsy to list items of personal importance in each HRQOL domain, rate their severity, and rate their own HRQOL relative to other people. This produced a profile of the difference between perceived and desired health status whose validity, reliability and responsiveness appear adequate. ${ }^{15}$ Although experience with this instrument is scarce, it demonstrates that discrepancy between perceived self-image and self-status is a powerful predictor of psychosocial well-being in epilepsy.

\section{HEALTH-RELATED QUALITY OF LIFE IN EPILEPSY}

Clinicians usually ask patients questions such as "How are you doing?" These questions incorporate much more than a simple seizure count, they capture the patients' subjective perception of their seizures, treatment efficacy and side-effects. ${ }^{16}$ The importance of measuring patient-centred health status and well-being in epilepsy resulted in the development of epilepsyspecific HRQOLinstruments and in a growing body of literature on this topic from 1990 onwards. In addition to generic HRQOL instruments such as the Sickness Impact Profile (SIP), ${ }^{17}$ Nottingham Health Profile (NHP) ${ }^{18}$ and Short Form-36 (SF$36),{ }^{19}$ clinicians now have access to a plethora of epilepsyspecific HRQOL tools, such as the closely related QOLIE- $89^{20}$ and ESI-55, ${ }^{1}$ and Liverpool Scales. ${ }^{4}$ Given this array of instruments, clinicians must decide which tool is best for their particular objective.

\section{Choosing The best instrument to measure HRQOL}

Choosing instruments to assess outcome should be carefully considered. Wrong choices may result in uninterpretable or irrelevant data and wasted efforts. Answering the following four questions should assist clinicians in choosing an appropriate HRQOL tool:

1) What is the scope of the intended HRQOL assessment? This can be limited to one or a few areas of interest or include all aspects of HRQOL.

2) What is the purpose of measuring HRQOL? Clinicians may wish to distinguish between patient groups at one point in time, to prognosticate outcome, or to measure the amount of change in response to an intervention.

3) How good are the available instruments? This refers to the instrument's content and measurement properties, ie., validity, reliability and responsiveness.

4) What are the practical issues in using an instrument? It is important to know its size, whether it is self- or intervieweradministered, whether training is required, and whether epilepsy patients can complete it.

\section{Scope of HRQOLinstruments}

Guyatt et $\mathrm{al}^{21}$ have proposed a taxonomy for HRQOL instruments based on their scope. Two main categories are recognized, generic and specific. Each has advantages and disadvantages.

\section{Generic or general instruments}

These cover all the recognized domains of HRQOL. The main advantages of generic tools are comprehensiveness, broad applicability (which allows comparisons across groups of patients and conditions), and knowledge about their measurement properties gained through extensive use in diverse populations. The major disadvantages are lack of focus on relevant aspects of specific conditions, and potentially an inferior ability to detect clinically important changes, although the latter remains to be proven.

Generic instruments consist mainly of health profiles and utility indexes. Examples of health profiles include the well known SIP, ${ }^{17}$ the NHP, ${ }^{18}$ and the SF-36. ${ }^{19}$ In addition, single-item scales asking "How is your quality of life?" meet the criteria for a generic instrument. ${ }^{22}$ Utility measures provide a single number 
describing the patients' valuation of their health status, ranging from 0 (death) to 1 (perfect health). Utility indexes are required for cost-utility analyses and for estimating quality adjusted life years (QALYs). The latter is a standard measure that incorporates quantity (time) and quality (HRQOL) of life, allowing for comparisons across conditions and interventions. QALYs are the current recommended standard for economic evaluations of medical interventions. ${ }^{23}$ Commonly used utility methods are the Standard Gamble, ${ }^{24}$ Time Trade Off, ${ }^{25}$ Utilities Index, ${ }^{26}$ Quality of Well Being Index ${ }^{27}$ and EuroQOL. ${ }^{28}$ For a complete treatment of utilities see Torrance. ${ }^{29}$

\section{Specific or targeted instruments}

By focusing on a single condition, patient, symptom, population or function, these tools may be more responsive to change than generic tools. However, this may come at the expense of decreased comprehensiveness, and inability to compare between conditions or programmes. Their usefulness in AED trials ${ }^{30}$ and epilepsy surgery has been proven. ${ }^{11,31}$ For instance, pain or mobility, commonly assessed by generic instruments, are of marginal importance to epilepsy patients. On the other hand, stigma and cognitive function, both important in epilepsy, are assessed by epilepsy-specific instruments. A combination of generic and specific instruments is probably a good compromise. This is accomplished in a closely related set of epilepsy-specific instruments, the QOLIE-8920 (and its abbreviated versions the QOLIE-31 $1^{32}$ and QOLIE-10 ${ }^{33}$ ) and ESI$55,{ }^{1}$ by adding epilepsy-specific items to a widely used generic instrument, the SF-36. ${ }^{19}$ In the Liverpool HRQOL model this is achieved by using a number of independent generic and epilepsy-specific scales as a battery. ${ }^{4}$

\section{Purpose of HRQOLinstruments}

Not all HRQOL instruments serve all purposes. Thus, one must define the clinical question in order to choose the best instrument to answer it. In general HRQOLis measured for three reasons: ${ }^{34}$

1) To distinguish between individuals or groups on an underlying dimension, eg., does HRQOL vary by type and severity of epilepsy. ${ }^{35}$ Instruments used for this purpose must have adequate discriminative properties.

2) To predict which patients will develop a specific outcome as compared to an available criterion standard, eg., the Karnofsky scale for cancer patients has important predictive usefulness. These tools must have proven predictive properties.

3) To measure change within patients over time, either as a result of natural history or clinical interventions, eg, is the amount of improvement in HRQOLafter epilepsy surgery substantial or clinically relevant? Such instruments must show adequate responsiveness. ${ }^{11}$

Ideally, an instrument should have demonstrated usefulness for all three functions. Formal research has shown that several of the generic tools have such versatility, eg., the SIP, ${ }^{17}$ the $\mathrm{NHP}^{18}$ and the SF-36. ${ }^{19}$ However, general statements such as "this is a validated instrument" are meaningless when choosing an instrument, unless information about validity, reliability and responsiveness is provided. Clinicians must keep in mind that ability to discriminate between patients groups does not guarantee ability to measure change over time. ${ }^{11}$ In epilepsy surgery, as in any other intervention, clinicians are interested in measuring within-patient improvement over time. Therefore, instruments used to assess outcome must have proven responsiveness. Epilepsy-specific instruments with demonstrated responsiveness include the Liverpool battery of instruments, ${ }^{36}$ ESI-55, ${ }^{11}$ and QOLIE-89. ${ }^{37}$

\section{Instrument content and measurement properties}

For a review of desirable measurement attributes of evaluative HRQOL instruments see McDowell, ${ }^{38}$ Nunnally, ${ }^{39}$ and Guyatt. ${ }^{40}$ In general, in addition to content validity (are the items relevant, comprehensive, and unambiguous), the evaluative instrument must show evidence of responsiveness. The latter assesses the signal-to-noise ratio in evaluative instruments, the same way reliability does so in discriminative instruments. The signal refers to true within-subject change over time, and noise denotes within-subject differences

Table: The choice of HRQOL tool depends on the study objectives, clinical circumstances and instrument measurement properties.

\section{Purpose}

Obtain a general health status measure:

Brief instruments

Longer instruments

Compare epilepsy with other diseases

Obtain QALYs (for cost-effectiveness studies)

Obtain epilepsy-specific health status measure:

Brief instruments

Longer instruments

Measure change in health status due to interventions or to evolution of disease

Measure seizure severity

Measure AED adverse events

Measure impact of epilepsy

\section{Candidate Instruments*}

\author{
SF-36, HUI-3, EuroQol, NHP \\ SIP \\ SF-36, SIP, NHP, HUI-3, EuroQol \\ HUI-3, EuroQol
}

Liverpool scales, QOLIE-31, QOLIE-10

QOLIE-89, ESI-55

QOLIE-89, ESI-55, SF-36, Liverpool scales

Liverpool scales, NHS scale

Liverpool scales

Liverpool scales

*See text for abbreviations and descriptions 
unrelated to true within-subject change. Responsiveness, therefore, refers to the instrument's ability to detect minimum clinically important differences in the condition of interest. Minimum clinically important difference may be defined as the change in health status perceived by the patient (or physician, depending on the viewpoint) to be of sufficient magnitude to require treatment, in the absence of unacceptable side effects. ${ }^{41}$ If responsiveness has not been established, at the very least there should be no ceiling or floor effects of the scores (score clustering at the extremes of the possible range), ie., they must be potentially changeable. ${ }^{40}$

\section{Viewpoint of HRQOL measurement}

The viewpoint in HRQOL studies refers to who interprets the patient's experience related to health. This is an important determinant in choosing a HRQOL instrument. The three major viewpoints frequently adopted in HRQOL assessment are the clinician's, the patient's, and less frequently society's, i.e., society attributes a certain value to a given health state. ${ }^{42}$ Clinicians are most interested in capturing the patient's subjective experience of a particular health state. Therefore, patient-centred measures are preferable.

A summary of recommended, validated HRQOL instruments is presented in the Table. These recommendations should be tempered by clinicians' preferences, as well as by the caveats described above regarding choice of instrument.

\section{Practical issues in USING HRQOL instruments}

\section{Respondent burden}

This refers to the time and effort required to complete one or a set of questionnaires. The problem is not inconsequential. Lengthy, difficult or ambiguous questionnaires are likely to be answered incompletely, carelessly or not at all. This results in unreliable data or in high rates of noncompliance. A similar problem may be encountered by administering questionnaires too frequently. The following are recommended:

1) Look for published evidence of respondent burden for candidate instruments, ie., time it takes to complete, and reported response rates.

2) Read the questionnaire and assess its content, clarity and size.

3) Select a feasible number of instruments.

4) Plan frequency of administration carefully in order to avoid noncompliance (too frequent) or missing a lot of information (not frequently enough). A reasonable minimum may be baseline and one pre-established follow-up.

5) Pretest the instrument set before administering it widely. This extra bit of initial work is worth the effort.

\section{Administering the instrument}

The manner in which instruments are used is no less important than their measurement properties. Inappropriate use of a good tool will produce uninterpretable or biased results. Unwary clinicians often incur the following errors:

\section{1) Modifying the questionnaire}

It is difficult for clinicians to resist the temptation to add, delete, or reword a questionnaire to their specific liking, and then analyse and report results as per the original. This is a dangerous practice. Unless the changed instrument is subjected to formal validation, one cannot be assumed that data obtained with it are equivalent to data obtained with the original version. Only two alternatives exist: a) Use the unmodified instruments, adding other tools to capture areas of interest as needed. b) Design and validate a new instrument, which is a labourious endeavour.

\section{2) Using the wrong administration technique}

Specific tools are designed for specific modes of administration. They may be strictly self- or intervieweradministered, and some can be used in either way. The developers' guidelines should be followed in this respect.

\section{3) Administering instruments in an inconsistent fashion}

Variations in mode and timing of administration introduces noise and bias. For instance, interviewer administration of selected patients may allow advertent or inadvertent coaxing by the interviewer (bias), or decrease precision of responses (noise). By the same token, all patients should receive the same amount of instruction. Similarly, variations in time of administration ignore the fact that HRQOL is not static, ie., it changes with time. ${ }^{43}$ If changes occur in a particular direction, eg., worsening with time, results may be biased or diluted by including early measurements. For example, reports stating that "HRQOL was measured one to two years after surgery" should be cautiously interpreted.

\section{REFERENCES}

1. Vickrey BG, Hays RD, Graber J, et al. A health-related quality of life instrument for patients evaluated for epilepsy surgery. Med Care 1992; 30: 299-319.

2. Gill TM, Feinstein AR. A critical appraisal of the quality of qualityof-life measurements. JAMA1994; 272: 619-626.

3. Cramer JA, Smith DB, Mattson RH, et al. A method of quantification for the evaluation of antiepileptic drug therapy. Neurology 1983; 33(Suppl 1): 26-37.

4. Baker GA, Smith DF, Dewey M, Jacoby A, Chadwick DW. The initial development of a health-related quality of life model as an outcome measure in epilepsy. Epilepsy Res 1993; 16: 65-81.

5. O'Donoghue MF, Duncan JS, Sander JW. The National Hospital Seizure Severity Scale: a further development of the Chalfont Seizure Severity Scale. Epilepsia 1996; 37: 563-571.

6. Lennox W, Lennox M. Epilepsy and Related Disorders. Vols. 1 and 2., Boston: Little, Brown 1960.

7. Taylor DC. The components of sickness: disease, illness, and predicaments. Lancet 1979; 1: 1008-1010.

8. Dodrill C, Batzel LW, Queisser HR, Temkin NR. An objective method for the assessment of psychological and social problems among epileptics. Epilepsia 1980; 21: 123-135.

9. Cramer JA. Quality of life for people with epilepsy. Neurol Clin 1994; 12(1): 1-13.

10. Trostle JA, Hauser WA, Sharbrough FW. Psychologic and social adjustment to epilepsy in Rochester, Minnesota. Neurology 1989; 39: 633-637.

11. Wiebe S, Rose K, Derry PA, et al. Outcome assessment in epilepsy: comparative responsiveness of quality of life and psychosocial instruments. Epilepsia 1997; 38: 430-438.

12. Chaplin JE, Yepez R, Shorvon S, Floyd M. A quantitative approach to measuring the social effects of epilepsy. Neuroepidemiology 1990; 9: 151-158

13. Hermann BP. Quality of life in epilepsy. J Epilepsy 1992; 5: 153165.

14. Chaplin JE, Yepez R, Shorvon SD, Floyd M. National general practice study of epilepsy: the social and psychological effects of a recent diagnosis of epilepsy. Br Med J 1992; 304: 1416-1418.

15. Kendrick AM, Trimble MR. Repertory grid in the assessment of 
quality of life in patients with epilepsy: the quality of life assessment schedule. In: Trimble MR, Dodson WE, eds. Epilepsy and Quality of Life. New York: Raven Press Ltd., 1994: 151-163.

16. Chadwick D. Measuring antiepileptic therapies: the patient vs the physician viewpoint. Neurology 1994; 44: S24-S28.

17. Bergner M, Bobbitt RA, Carter W, Gilson BS. The sickness impact profile: development and final revision of a health status measure. Med Care 1981; 19: 787-805.

18. Hunt SM, McEwen J, McKenna SP. Measuring health status: a new tool for clinicians and epidemiologists. J Roy Coll Gen Pract 1985; 35: 185-188.

19. Ware JE, Jr., Sherbourne CD. The MOS 36-Item Short-Form Health Survey (SF-36): I. Conceptual Framework and Item Selection. Med Care 1992; 30: 473-482.

20. Devinsky O, Cramer JA. Assessing quality of life in epilepsy: development of a new inventory. Epilepsia 1993; 34(Suppl 4): S1-S44

21. Guyatt GH, Veldhuyzen Van Zanten SJO, Feeny DH, Patrick D. Measuring quality of life in clinical trials: a taxonomy and review. Can Med Assoc J 1989; 140: 1441-1448.

22. Guyatt G, Patrick D, Feeney D. Postscript. Control Clin Trials 1991; 12(Suppl 4): 266-269.

23. Weinstein MC, Siegel JE, Gold MR, Kamlet MS, Russell LB. Recommendations of the panel on cost-effectiveness in health and medicine. JAMA1996; 276: 1253-1258.

24. Gafni A. The Standard Gamble method: what is being measured and how it is interpreted. Health Serv Res 1994; 29: 207-224.

25. Torrance GW. Utility approach to measuring health-related quality of life. J Chronic Dis 1987; 40: 593-603.

26. Torrance GW, Feeny DH, Furlong WJ, et al. Multi-attribute utility function for a comprehensive health status classification system. Health Utilities Index Mark 2. Med Care 1996; 34: 702-722.

27. Kaplan RM, Bush JW. Health-related quality of life measurement for evaluation research and policy analysis. Health Psychol 1982; 1: $61-80$.

28. Brazier J, Jones N, Kind P. Testing the validity of the Euroqol and comparing it with the SF- 36 health survey questionnaire. Qual Life Res 1993; 2: 169-180.

29. Torrance GW. Measurement of health state utilities for economic appraisal: a review. J Health Economics 1986; 5: 1-30.
30. Wagner AK, Keller SD, Kosinski M, et al. Advances in methods for assessing the impact of epilepsy and antiepileptic drug therapy on patients' health-related quality of life. Qual Life Res 1995; 4: 115-134.

31. Vickrey BG, Hays RD, Engel J, Jr., et al. Outcome assessment for epilepsy surgery: the impact of measuring health-related quality of life. Ann Neurol 1995; 37: 158-166.

32. Cramer JA, Perrine K, Devinsky O, et al. Development and crosscultural translations of a 31-item quality of life in epilepsy inventory. Epilepsia 1998; 39: 81-88.

33. Cramer JA, Perrine K, Devinsky O, Meador K. A brief questionnaire to screen for quality of life in epilepsy: the QOLIE-10. Epilepsia 1996; 37: 577-582.

34. Kirshner B, Guyatt GH. A methodologic framework for assessing health indices. J Chronic Dis 1985; 38: 27-36.

35. Jacoby A. Epilepsy and the quality of everyday life. Findings from a study of people with well-controlled epilepsy. Soc Sci Med 1992; 34: 657-666.

36. Baker GA, Jacoby A, Smith D, et al. Quality of life in epilepsy: the Liverpool initiative. In: Trimble MR, Dodson WE, eds. Epilepsy and Quality of Life. New York: Raven Press Ltd., 1994: 135-150.

37. Wiebe S, Derry PA, Rose K, McLachlan RS. In search of a minimum clinically important change in epilepsy-specific quality of life instruments. Epilepsia 1997; 38: S83-S84.

38. McDowell I, Newell C. Measuring Health: A Guide to Rating Scales and Questionnaires. New York: Oxford University Press 1987; 12-31.

39. Nunnally JC. Psychometric Theory. New York: McGraw-Hill 1978.

40. Guyatt GH, Walter S, Norman G, Guyatt G. Measuring change over time: assessing the usefulness of evaluative instruments. J Chronic Dis 1987; 40: 171-178.

41. Jaeschke R, Singer J, Guyatt GH. Measurement of health status. Ascertaining the minimal clinically important difference. Control Clin Trials 1989; 10: 407-415.

42. Guyatt GH, Feeney D, Patrick D. Issues in quality of life measurement in clinical trials. Control Clin Trials 1991; 12: 81S$90 \mathrm{~S}$.

43. Wiebe S, Derry P, Gilmore B, Foster-Janzen L. Does the time frame of quality of life (QOL) questionnaires affect the results? Epilepsia 1997; 38: S135. 